Splenomeg. Americ. Journ. of the med. sc. 1905. Zit. nach $\mathrm{S} \mathrm{chl}$ a ge $\mathbf{n}$ ha u f er. - 16. Harris u. Herzog, Splenectomie bei Splenomeg. primit. D. Ztschr. f. Chir. 1901, LIX. - 17. Do lli n g e r, Az anaemia splenica v. splenomeg. primitiva. Orvosi Hetilap, 1902, 18-19 (ungarisch). - 18. B o r is sow a, Beitr. z. Kenntn. d. B a n t i schen Krankh. u. Splenomeg. Virch. Arch. Bd. 172. - 19. S c h l ag e $n$ h a u f e r, Utb. meist famil. vorkom. histolog. charakt. Splenomegalieen (Typus G a u c ber). Virch. Arch. Bd. 187. 20. M a x i m o w, Ưb. entzündl. Bindegewebsneubild. b. Axolotl. Z i e g l e r s Beitr. Bd, XXXIX. - 21. A $1 \mathrm{~m} \mathrm{k}$ v is $\mathrm{t}$, Ủb. d. Emigrationsfähigk. d. Lymphozyten. Virch. Arch. Bd. 169. - 22. N e u m a n $n$, Hämatolog. Studien. Virch. Arch. Bd. 174. - 23. P a p p e n h e im, Atlas d. menschl. Blutzellen. 1905. - 24. S c h rid d e, Die Wanderungsfähigh. d. Lymphozyten. Münch. med. Wschr. 1905, 39. - 25. D e r s el b e, Utb. d. Wanderungsfähigk. d. Plasmazellen. Verhandl. d. D. Path. Ges. 1906. - 26. O r th, - S p e r o n i, Arb. a. d. Path. Inst. z. Berlin. Festschr. 1906. S. 160.

\title{
VII.
}

\section{Multiples diffuses Myelom (Myelomatosis ossium) mit reichlichen Kalkmetastasen in die Lungen und andere Organe.}

(Aus dem Peter-Paul-Stadt-Krankenhaus zu St. Petersburg.)

Von

Privatdozent Dr. Th. Ts eh is tow itseh und Dr. Helene Kolessnik off.

(Hierzu 5 Textfiguren.)

Im Jahre 1873 hatte $\mathrm{R} u$ s t i z k y eine eigenartige Erkrankung des Knochenmarks beschrieben, welche die Bildung mehrerer - bis ungefähr acht - Tumoren in den Knochen des Rumpfes, ebenso im Schädel und Humeri, darbot, die ausschließlich von Knochenmarkselementen identischen Rundzellen gebildet wurden. Obgleich diese Tumoren Sarkomen sehr ähnlich waren, unterschieden sie sich doch von den letzteren dadurch, daß sie aus Knochenmarkzellen bestanden, nur im Knochenmark saßen und nirgends zu Metastasen geführt hatten. Deswegen schlug $\mathrm{R} \mathrm{u}$ sti z k y vor, solche Knochenmarkstumoren Myelome zu nennen; $R u$ stizky hielt sie für nicht bösartige Tumoren, oder lokale knotige Knochenmarkshyperplasien, da sie zwar in mehreren Knochen gleichzeitig auftreten, aber keine Metastasen bilden.

Rustizkys Befund, der ziemlich lange ohne gebührende Beachtung gelassen wurde, fand später Zustimmung von seiten 
vieler Forscher, die den Begriff „Myelom“ erweitert und präzisiert hatten. Jetzt wišen wir, daß diese 'Tumoren gewöhnlich Männer von über 40 Jahren befallen und vornehmlich das Knochenmark des Rumpfskeletts und des Schädeldaches affizieren; sie verursachen heftige Schmerzen besonders bei Druck; sie treten entweder als multiple Knoten auf, oder als diffuse Hyperplasien des gesamten Knochenmarks; durch diese Tumorbildungen werden die Knochentrabekeln der Spongiosa so gut wie vollständig zum Schwunde gebracht und dadurch teils Osteoporose und Knochenbrüchigkeit, besonders der Rippen, bedingt, teils Deformitäten der Wirbelsäulc und des Brustkorbes erzeugt; Metastasen in andere Organe werden niemals beobachtet; dagegen führt die Tumorbildung zu sekundärer Anämie und zur Ausscheidung durch den Harn von einer Art Albumose, welche von $K \ddot{~ u h ~} \mathrm{n}$ e und $\mathrm{K}$ a h l e r genau studiert wurde und den Namen ihres Entdeckers B e n c e J o h n e s erhalten hat.

Nach dieser kurzen Skizze des Myelombegriffes gehen wir zu dem von uns beobachteten Fall über.

Krankengeschichte. (Fräul. Dr. H. Kolessnikoff). Am 19. November 1907 wurde ins Peter-Paul-Stadt-Krankenhaus von St. Petersburg die Bauersfrau des Gouvernements Pskoff Cath. P. aufgenommen, 36 Jahre alt, verheiratet, welche über starke Schwäche, Banchschmerzen, Erbrechen, Obstipation, Schmerzen ịn Rücken, der Brustgegend, Extremitäten und über Husten klagte. Die Kranke gehört zu einer armen Familie; ihr Vater litt an Alkoholismus und starb 46 Jahre alt geisteskrank; die Mutter, 60 Jahre alt, lebt gesund. Von den 7 Kindern starb eine Schwester an Uteruskrebs; eine zweite ist verheiratet und sehr krank; die übrigen Brüder and Schwestern sind gesund. 21 Jahre alt wurde die Kranke eine Prostituierte und gebar bald einen Knaben, der jetzt 14 Jahre alt und gesund ist; später wurde sie syphilitisch und einer merkuriellen Behandlung im Kalinkinschen Stadtkrankenhause unterworfen. Im Jahre 1903 wurde sie auf 8 Tage ins Alexander-Stadtkrankenhaus aufgenommen mit erhöhter $\mathrm{t}^{0}\left(38.5^{\circ}\right)$ und Klagen über Schwäche and Kopfschmerzen. Die Diagnose lautete Influenza. Der Harn war damals eiweißfrei. Vor 5 Jahren hatte sich die Kranke verheiratet und erhielt mehrmals Prügel von ihrem Manne auf den Kopf und den Rumpf. Die Ehe blieb kinderlos. Sie fühlt sich unwohl schon ungefähr 5 Jahre; erst traten Schmerzen im Nacken, in der Brust, im Rücken und in den Extremitäten, besonders während der Nacht auf; es entwickelten sich Schwäche, Anämie und Herzklopfen. Vor 2 Jahren pflegten stechende Schmerzen in den Beinen immer öfter aufzutreten, von einem Lähmungsgefühl begleitet. Seit 6 Monaten trat Atemnot, Erbrechen und Obstipation auf. Vom 17. Nov. nach einem Diätfehler, fing die Kranke

Virehows Archiv f. pathol. Anat. Bd. 197. Hft. 1. 
an über heftige Magenschmerzen zu klagen, erbrach mehrmals und legte sich ins Bett ohne mehr aufzustehen.

S t a t. praes e n s. Die Patientin ist in schwer krankem Zustande, stark abgeschwächt, spricht kaum mit leiser Stimme, Atmung stark beschleunigt (bis 40 in d. M.), oberflächlich, unter Teilnahme der Nasenflügel. Sie ist mittelgroß, von regelmäßigem Körperbau, schwacher Ernährung; Haut und Schleimhäute blaß, Brustkorb flach, Supra- und Infraklavikulargruben tief eingesunken, Lymphdrüsen nicht vergrößert; $t^{0} 36,6^{\circ}$, P. 112 regelmäßig, schwach gespannt, Zunge stark belegt; Lungengrenzen normal. Atemgeräusch abgeschwächt, rauh; Herzgrenzen normal, über der Art. pulmonalis schwaches systolisches Geräusch. Bauch nicht aufgetrieben, im Dickdarm Fäkalanhäufungen wahrnehmbar. Leber und Milz nicht vergrößert; die gynäkologische Untersuchung negativ; Harn (bis auf 700,0 gesammelt) trüb, alkalisch, mit großem weißlichem Niederschlage, der aus phosphorsaurem Ammoniak-Magnesium, amorphen Phosphaten und kohlensaurem Kalk bestand; außerdem Plattenepithel, einige hyaline Zylinder und Leukozyten. Sp. G. 1,010, Eiweib) bis 2,5\% (nach E s b a c h ). Körpergewicht: 49,3 am 25. XI. - 47,2. Weitere Gewichtsaufnahmen wurden unmöglich.

Krankheits ver la uf. Vom 23. Nov. stellten sich Temperaturerhöhungen bis $38,6^{\circ}$ ein, dann wurden krepitierende Blasengeräusche sub spina scapulae d., Dämpfung in rechter Lungenspitze, verstärktes Vibrieren und bronchialer Charakter des Atemgeräusches unter der rechten Klavikula und rauhes abgeschwächtes Atmen an anderen Stellen und Rasselgeräusche konstatiert. Vom 28 an erschien noch Dämpfung auch links unten, laute Krepitation und Bronchialatmen und schleimig-eitriger dicker schaumiger Auswurf, der keine Kochschen Bazillen enthielt. Dyspnoe noch mehr ausgesprochen. Später wurde vom pneumonischem Prozesse auch der linke Oberlappen befallen, indem Auflösung nirgends boobachtet wurde. Ungefähr gegen diese Zeit war seitens des Harns eine sehr wichtige Tatsache bemerkt worden, die leider außer Acht gelassen wurde: der weiße Ring, der an der Berührungsstelle mit Salpetersäure entstand, verringerte sich bemerkbar, löste sich sogar fast vollständig auf beim Aufkochen des Harns, um beim Abkühlen wieder anszufallen. Das Aufkochen mit Essigsäure rief keinen Niedersehlag hervor, indem mit E s b a c h schem Reagens immer größere Quantitäten Eiweiß, bis auf $6 \%$, ausgefällt zu werden pflegten. Diese Reaktionen sind für $\mathrm{B}$ e n c e - J o h n e s sche Albumose ganz charakteristisch. Im weiteren Verlauf der Krankheit bei dawernden Erscheinungen seitens der Lungen wurde die Dyspnoe immer quälender (bis 52 pro Min.), der Kräfteverfall nahm zu, es trat Zyanose und Trübung des Gedächtnisses ein, und am 25. Tage des Aufenthaltes im Krankenhause, am 12. Dez. früh, trat Exitus letalis ein.

Sektionsprotokoll (Privatd. Dr. Th. Tschistowitsehi). Stark abgemagerte Leiche von mittlerer Größe und sehr schwachem Körperbau, Hautdecken sehr blaß; Füße, besonders links, leicht ödematös.

Beide Lungen frei, sehr groß und auffallend schwer. Pleuraoberfläche überall glänzend. Lungengewebe ausgesprochen hart, Schnittoberfläche grau- 
weißlich, trocken, fast kaseös, rauh, stellenweise pastös, stellenweise, namentlich in rechter Spitze, fast holzderb; doch seheinen die Alveolen sogar in den härtesten Stellen in ihrer Mehrzahl leer zu sein, und die Erhärtung hauptsächlich zwischen den Alveolen, in den Septen ihren Sitz zu haben. Beim Schneiden des Lungengewebes oder beim Abschaben der Schnittoberfläche mit dem Messer wird ein eigenartiges Knistern wahrgenommen. Die Bronchien, abgesehen von einer mäBigen Hyperämie, ohne Besonderheiten.

Herz 91/2-91/2, Herzfleisch ziemlich derb, die Spitzen der Papillarmuskeln weißlich, hart, knistern beim Aufschneiden; Aortaklappen mit Kalk imprägniert, rigid, übrige Klappen ohne Besonderheiten.

Milz $101 / 2-6$, Gewebe blutarm, bräunlichrosa, ziemlich derb, Pulpa wird spärlich abgeschabt.

Leber 28-20-13-6, Gewebe bräunlich, Schnittoberfläche glänzend, Zeichnung gut ausgesprochen. Die Gallenblase enthält viel dicke grüne Galle.

Nieren grob, Kapseln leicht abziehbar, Oberfläche glatt, Rindenschicht von weiblicher Farbe mit weißlich-gelblicher Streifung, weicher Konsistenz, glänzend. Nierenbecken ohne Besonderheiten.

Im hinteren Douglasraum einige fibröse Verwachsungen. Uterus und Ovarien sonst ohne Besonderheiten.

Die Falten der Magenschleimhaut sind sehr dick und derb anzufühlen. Dünndarm ohne Besonderheiten; die Schleimhaut des Dickdarms etwas hyperämisch.

Gehirnhäute und Gehirn zeigen außer Anämie nichts Abnormes.

Sämtliche Rumpflmochen (Rippen, Brustbein, Schlüsselbein, Darmbein, Wirbel) sind sehr brüehig, zerbrechen bei geringer Gewalt oder werden mit eigenartigem Knistern leicht eingedrückt; ihre Spongiosa ist rarefiziert, stellenweise ganz geschwunden, die Kortikalis bedeutend verdünnt; das Knochenmark erscheint vermehrt, graurötlich, saftig, weich und homogen. (Die langen Röhrenknochen konnten leider nieht untersucht werden).

$\mathrm{Epikrisis.} \mathrm{Myelomatosis} \mathrm{diffusa} \mathrm{et} \mathrm{halisteresis} \mathrm{ossium.} \mathrm{Calcinatio}$ pulmonum. Pneumonia catarrh. chronica et interstitialis recens bilat. Calcinatio valvularum aortae et musculorum papillarium cordis, renum et parietum ventriculi. Nephritis chronica parenchymatosa.

Wir haben es also mit einer eigenartigen Erkrankung der Knochen des Brustkorbes und des Beckens bei einer nicht alten Frau zu tun, die zu schneller Osteoporose - Schwund der Spongiosabalken, - geführt hat und bemerkbare Knochenbrüchigkeit zu Folge hatte. Die Einschmelzung des Knochengewebes, besonders in den Rippen, wurde durch Umwandlung des Knochenmarkes in eine weiche graurote geschwulstartige Masse hervorgerufen; die Geschwulstmassen haben nirgends eine Knotenform angenommen, sondern ganz diffus in der Mehrzahl der flachen 
Rumpfknochen, ja vielleicht auch in den Röhrenknochen der Extremitäten, das normale Knochenmark ersetzt. Diese Umwandlung des Knochenmarks wurde von Ausscheidung großer Mengen $\mathrm{Bence}-\mathrm{J}_{0} \mathrm{~h} \mathrm{n}$ es schen Körpers (Albumose) im Harn begleitet, sie entspricht also derjenigen Krankheitsform, deren

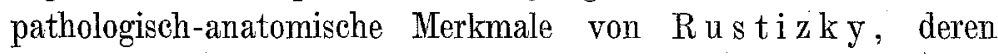
klinisches Bild von $\mathrm{Kahl}$ er geschildert wurden, weswegen die Myelomatose der Knochen den Namen "Ka hlers Krankheit" erhalten hat.

Die mikroskopische Untersuchung (Th. Tschistowitsch) hat die von uns aufgestellte Hypothese, daß es sich um einen Fall der $\mathrm{Ru}$ s ti zky - K a h l e r schen Krankheit, um multiples Myelom der Knochen handelte, vollständig bestätigt.

Die von der Schnittoberfläche der indurierten Lungengewebspartien abgeschabten Massen bestehen bei mikroskopiseher Untersuchung aus glänzenden stark lichtbrechenden strukturlosen Schollen, die bei Zusatz von Salzsäure sich ohne Gasbildung lösen, bei Zusatz von Schwefelsäure sich in zahllose Gypskristalle umwandeln. Es erwiesen sich also die grauweißen Massen, welche dem Lungengewebe eine so ungemein derbe Konsistenz verliehen, als Kalksalze und zwar aussehlieBlich als phosphorsaurer Kalk. Zur quantitativen Bestimmung der Kalkmengen in verschiedenen Organen und Knochen wurden von Fräulein H. Koles s n ik of f chemische Analysen ausgeführt, deren Resultate weiter unten zusammengestellt sind.

Zur mikroskopischen Untersuchung wurden Stücke von Organen in 0 r th scher Flüssigkeit, Spiritus und $4 \%$ Formollösung fixiert, Rippenstücke in $\mathrm{H}$ a u g sehem Salpetersäure-Alkoholgemisch dekalziniert und Zelloidin- sowie Paraffineinbettung angewandt. Die Resultate der Untersuchung der Schnitte werden nur in Kürze hier angeführt.

$\mathrm{R}$ i $\mathrm{p}$ p e $\mathrm{n}$. Schon bei geringer VergröBerung ist deutlich, daß die Rippen tiefgreifende Veränderungen eingegangen sind. Die Spongiosabalken sind meistens so gut wie restlos zugrunde gegangen. Die Kortikalis der Rippen ist sehr verschmälert, stellenweise unterbrochen; die innerhalb der Rippen gebildeten Hohlräume sind von einem Gewebe eingenommen, das ausschließlich aus einander gleichen, dicht zusammengedrängten Zellen besteht. Diese Zellen erinnern nach ihrer Größe beim ersten Anblick an große Lymphozyten; sie sind rundlich, polygonal (wegen gegenseitigen Drucks), besitzen einen reichlichen Protoplasmasaum, der fast homogen (ohne dentliche Körnung), mit Eosin schwach, mit Methylenblau ziemlich stark färbbar ist. Die Kerne liegen entweder zentral oder exzentrisch und sind von verschiedener Größe: die einen sind sehr klein, färben sich diffus und sehr stark; wie diejenigen der Erythroblasten; die anderen gleichen vollkommen Lymphozytenkernen; die dritten endiich sind voluminös, hell, fast bläschenförmig mit deutlichem zartem Chromatinnetz und 
großem Kernkörperchen; doch zeigen die Chromatinmassen keine für Plasmazellen charakteristische Lagerung. Diese drei Abarten derselben Zellenelemente liegen dicht nebeneinander ohne bemerkbare Zwischensubstanz, stellenweise sind sie in Reihen oder radiären Streifen angeordnet; zwisehen den Zellengruppen verlaufen spärliche Bindegewebsbüschel, die nur unter dem Periost und neben Knochenbalken eine starke Entwicklung erreichen. Im ganzen ist der Gewebsbau den Rundzellensarkomen ähnlich: die Zellenreihen schieben sich zwischen die Bindegewebsfasern hinein, indem sie dieselben verdrängen und allseitig die Knochenbalken umsehließen; die letzteren erscheinen an ihrer Oberfläche wie angefressen, doch enthalten deren Ausbuchtungen sehr wenig Osteoklasten, sondern dieselben Tumorzellen, welche das Knochenmark ersetzt haben. Die äußere Oberfläche der Knochenbalken ist stellenweise von Osteoblastenreihen unasäumt. An jenen Stellen, wo die Knochenrindenschicht Unter-

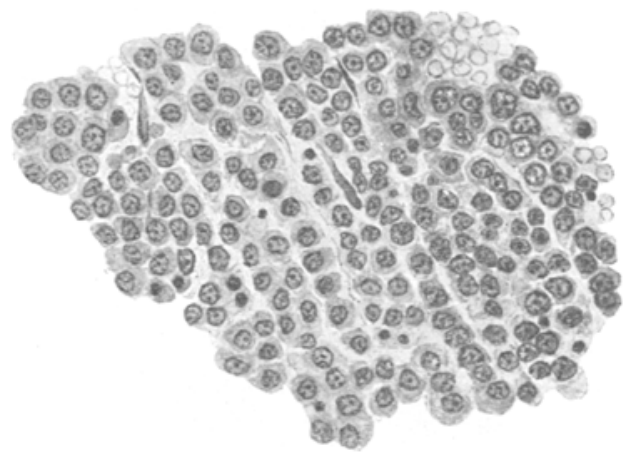

Fig. 1.

Knochenmark einer von Myelomatose betroffenen Rippe: Das Gewebe besteht aus ganz einander gleichen, bald größere blasse, bald kleine stark tingierte Kerne enthaltenden Myeloblasten Obj. DD, Okular 3. Vergr. 326fach.

brechungen (Lücken) zeigt, drängen sich die Tumorzellen bis ins Periost hindurch und können sogar mitten im Fettgewebe zwischen den Muskelbündeln getroffen werden; doch erreicht diese Verbreitung der Zellen keine bedentenden Dimensionen und überschreitet nirgends die unmittelbare Nachbarschaft der Rippenknochen; Metastasen oder Disseminationsknoten werden nicht gebildet.

Außer der beschriebenen Zellenart begegnet man im Knochenmarkraum gewöhnlich keinen anderen normalen Zellformen: Fettzellen fehlen, Megakaryozyten kommen nur selten zum Vorschein, dabei haben sie die Gestalt mehrkerniger Riesenzellen angenommen; mehrkernige Leukozyten und deren Vorstufen (Myelozyten), sowie Erythroblasten (kernhaltige rote Blutkörperchen) werden ganz und gar vermibt; hier und da kommen nur vereinzelte gekörnte eosinophile Zellen zum Vorschein; kurz und gut, es ist das normale Knochenmark vollständig durch ein neues Gewebe verdrängt, das aus eigenartigen einander ganz ähnliehen Zellen besteht; diese zeigen eine so reichliche 
Vermehrung, daß sie die Mehrzahl der Knochenbalken zur Atrophie geführt haben. Gefäße sind dünnwandig und verlaufen in geringer Zahl zwischen den Zellenmassen. Stellenweise kommen rote Blutkörperchen anseheinend frei neben den Tumorzellen vor.

Um die Natur der Tumorzellen näher festzustellen, wurden die Schnitte mit Triazidmischung nach Ehrli $\mathrm{ch}$ und mit polychromem Methy'enblau nach U n n a gefärbt. Dabei ergab sich, daß die Zellen weder neutrophile, noch basophile Körnung enthalten (eosinophile Zellen sind sehr spärlich zerstreut). Der Merkmale der Plasmazellen entbehren sie ebenfalls: zwar werden sie dureh

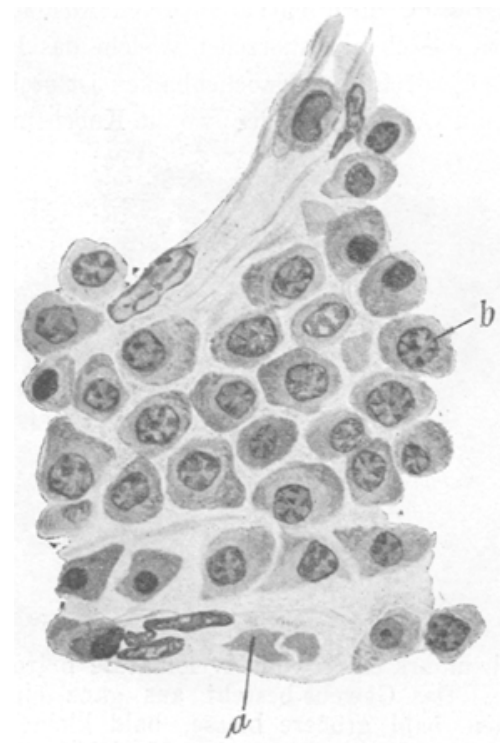

Fig. 2.

Dasselbe Gewebe bei stärkerer (730facher) Vergr. a Kapillare mit roten Bluttö̈rperchen; b Tumorzellen (Myeloblasten). Obj. Immers. Okular 3.

Methylenblau tingiert, doch vermiBt man den hellen Hof neben den Kernen, die dabei keine "Radkerne" sind. Aus allen diesen Angaben ist der Schluß zu ziehen, daß die Neubildung aus Elementen, die nahe Beziehung zu Myeloblasten (unreifen Myelozyten) haben, d. h. aus undifferenzierten sich kaum von den großen Lymphozyten unterscheidenden Knochenmarkzellen besteht. Die oben erwähnten Zellen, die sehr kleine sich intensiv färbende Kerne besitzen, sind als derartige Myeloblasten zu deuten, welche neuerdings von $M$ e $\mathrm{n} n \mathrm{e}$ unter dem Namen ,,kleinkernige Myeloblasten Engels" abgesondert und beschrieben wurden.

Die Verdrängung des Knochenmarkes durch die Tumorzellen (Myeloblasten) geht ganz diffus vor sich, doch ist sie nicht allenthalben abgeschlossen: 
hier und da kann man zwischen den Tumormassen noch inselartige kleine Uberreste des Knochenmarks mit seinen Fettzellen, Myelozyten, Megakaryozyten, polymorphkernigen Leukozyten und roten Blutkörperchen, bemerken; doch ist die Zahl dieser Inseln schon sehr gering.

L u n g e n. In Schnitten, die mit Hämatein (Hämatoxylin) gefärbt sind, kommt bei schwacher Vergrößerung ein regelmäßiges Netzwerk dunkelblauer Trabekel, die Alveolenwandungen entspreehen, zum Vorsehein. Dieses Netz ist durch reichliche Kalkablagerungen bedingt, die sämtliche Gefäßearterien, Venen und Kapillaren durchtränkt haben. An Querschnitten umgibt die Kalkmasse das Gefäß mit dickem Ring, der nach außen von der Endothelschicht

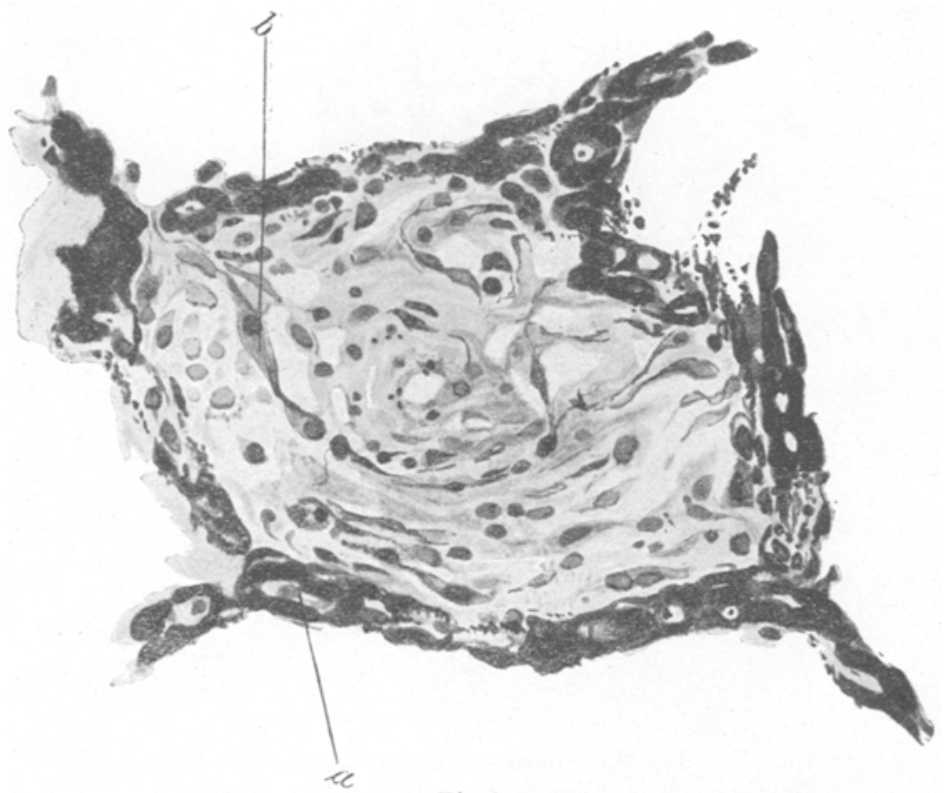

Fig 3.

Lungenalveole von jungem Granulationsgewebe erfüllt. Die Gefäße der Alveolenwand (a) sind mit Kalksalzen durchtränkt; b Fibroblasten. Vergr. 240fach.

gelagert ist; diese selbst bleibt unversehrt. Die Kalkniederschläge, teils als gekörnte Massen, teils als dicke unregelmäßige, zuweilen in plumpe Stücke zerfallene Balken erscheinend, nehmen auch das elastische Gewebe zwischen den Alveolen ein. Die GefäBlumina enthalten noch rote Blutkörperchen. Was die Alveolen selbst betrifft, so sind sie fast überall (in den affizierten Lungenpartien) durch junges hineinwachsendes saftiges durehsichtiges, ovale Kerne enthaltendes Bindegewebe erfüllt (spindelförmige Fibroblasten und Bindegewebsfaserbündel). Diese Verhältnisse treten besonders deutlich hervor an nach v a n G i e s o n gefärbten Präparaten. In anderen Alveolen sind außerdem noch Leukozytenansammlungen und große einkernige körner- und pigmenthaltige Zellen (,katarrhalische Zellen"), sowie rote Blutzellen zu bemerken. 
Es liegt also eine frische interstitielle Pneumonie vor, die von massenhafter Kalkablagerung in Gefäßwände, elastische Alveolensepten und bindegewebige Trabekeln begleitet wurde. Die Alveolenlumina sowie das diese ausfüllende junge Bindegewebe sind davon vollständig verschont geblieben.

$\mathrm{N}$ i e r e n. Die gewundenen Harnkanälchen zeigen keine bemerkbaren Veränderungen, ihr Epithel ist gut erhalten; dagegen ist es in $\mathrm{H}$ e $\mathrm{nl}$ e schen Schleifen hier und da desquamiert und liegt frei im Lumen derselben; auBerdem begegnet man hier und in den Tubuli recti einigen hyalinen Zylindern.

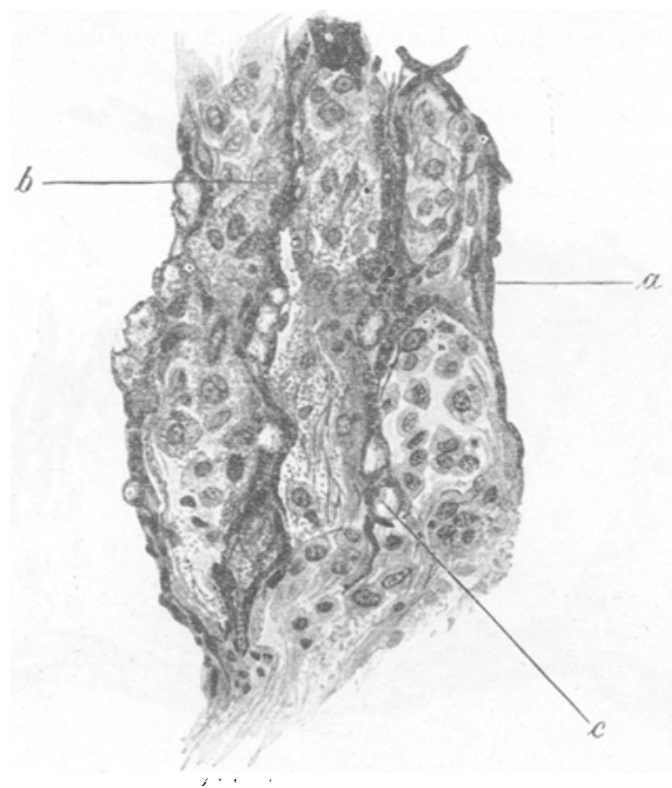

Fg. 4.

Fin Teil der Magenmukosa mit reicblicher Kalkablagerung in den Membranae propriae der Drüsen (a), den ${ }_{i}$ Kapillarwandungen (c) und Kalkkörnchen im Lamen der Drüsen.

Das Epithel der $\mathrm{B}$ o.w $\mathrm{m}$ a $\mathrm{n}$ schen Kapseln ist einreihig, deren Lumina enthalten kein Exsudat. Die Glomerulusschlingen, Membranae propriae der Kanälchen und Kapseln sind stellenweise mit Kalk imprägniert; Schollen und Ballen desselben sind auch frei in den Kanälchen angehäuft, besonders in den Tubuli recti; man findet solche Schollen, doch selten, auch zwischen den Kanälehen; dagegen sind die Gefäßwände, besonders solche großen Kalibers, von Kalkablagerungen frei, nur sind einzelne Vasa afferentia der Knäuel davon durchtränkt. Im ganzen sind die Kalkablagerungen in den Nieren ziemlich reichlich. Das Bindegewebe der Nieren scheint keine Zunahme erfahren zu haben, es bleibt also nur übrig, daß der weißliche Farbenton der Rindenschicht, der bei der Sektion an parenchymatöse Nephritis zu denken berechtigte, hauptsächlich 
durch reichliche Kalkablagerungen bedingt wurde, indem die Veränderungen im Parenchym sich als sehr gering erwiesen und nur die $\mathrm{H}$ e $\mathrm{n}$ l e schen Sehleifen und Tubuli recti betroffen haben.

M a g e n. Die Derbheit und Konsistenz der Schleimhautfalten ist dureh reichliche Kalkinkrustation der mittleren und submukösen Schleimhautpartien bedingt, namentlich der Membranae propriae der Pepsindrüsen, und der zwischen denselben verlaufenden kleinen Gefäßehen, sowie auch durch Verkalkung der Arterien und Venen der Submukosa und Muskularis. Die Kalkringe nehmen die Intima und Media ein, während die Endothelschicht davon frei bleibt; die verkalkten Gefäße sind bluthaltig. AuBer diesen Kalkablagerungen sind sonst keine Veränderungen in der Magenwand zu notieren.

Milz. Kalkablagerungen in Form regelmäBiger violetter Ringe finden sich in der Mehrzahl der Gefäße, zwischen Endothel und Muskularis. Das Milz-

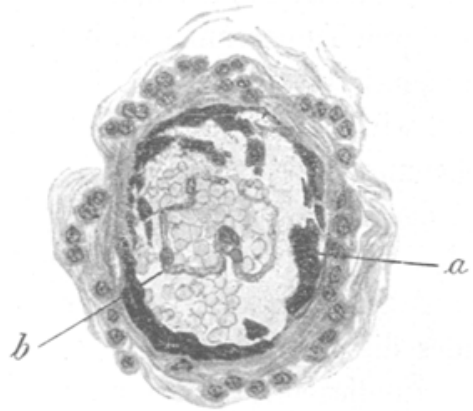

Fig. 5.

Reichliche Kalkablagerung in der Arterienmedia eines Milzgefäßes (a); die abgefallene Endothelschicht liegt frei im Gefäßlumen Vergr. 240.

parenchym zeigt keine Besonderheiten, nur sind die $\mathrm{M} \mathrm{a} \mathrm{l} \mathrm{p}$ i g h i schen Körperchen gering und spärlich; Bindegewebe nicht vermehrt, Gefäßwände nicht verdickt.

I m D i c k d a r m finden sich die Kalkablagerungen in den Gefäßwänden der Muskularis und der Subserosa, hauptsächlich in großen Arterien, zwischen Intimaendothel und Media. Die Sehleimhaut selbst samt ihren Schleimdrüsen ist frei von Kalkablagerungen.

In $d$ er L e b e r begegnet man nur selten Kalkablagerungen in den Wänden der Art. hepatica, wo sie denselben Platz wie in anderen Organen innehaben. Die Leberzellen sind verkümmert, stellenweise vakuolisiert; ihre Kerne verschieden groß, gut färbbar.

Im M y $\mathrm{k}$ a $\mathrm{rd}$ sind nur geringe ringförmige Kalkablagerungen in einigen Gefäßwänden zu sehen. Zum Schluß ist noch zu bemerken, daß in den Arterien, die Kalkablagerungen enthalten, keine entzündlichen Veränderungen der Wände (Verdickung der Intima, hyaline Degeneration usw.) gefunden wurden. 
Es liegt also ein Fall von allgemein in den Knochen des Rumpfes verbreiteter Vermehrung einer bestimmten Art Zellen vor, die ihren morphologischen Eigenschaften nach primären Myeloblasten am nächsten stehen, $d$. h. denjenigen Zellen des Knochenmarks, welche in ihrem Protoplasma neutrophile Körnung ausarbeiten und bei gewöhnlichen Umständen zu typischen Myelozyten werden. Die Vermehrung der Myeloblasten ging so schnell vor sich, daß sie allmählich ïberall die anderen Knochenmarkselemente verdrängt haben, die Spongiosabalken zum Schwund brachten und endlich, nashdem die Knochenschale stellenweise usuriert wurde, nach außen ins Periost und sogar jenseits desselben sich verbreiteten. Wäre die Kranke länger am Leben geblieben, so wäre es wahrscheinlich zu spindelförmigen durch Tumorenmassen bedingten Auftreibungen der Rippen gekommen, ja bis zu Rippenbrüchen; kurz es unterliegt keinem Zweifel, daß wir mit einem multiplen Myelom, resp. Myelomatose des Knochenmarks zu tun haben.

Die Übersicht der über Myelome in diesen letzten Jahren erschienenon ziemlich umfangreichen Literatur zeigt, daß verschiedene Verfasser in ihrer Auffassung der Natur und des pathologischanatomischen Bildes dieser Neubildungen sehr weit von einander abweichen. Die grundlegende Auffassung (Charakteristik) der Myelome, als einer multiplen, ausschließlich das Knochenmark affizierenden und niemals in anderen Organen Metastasen produzierenden Neubildung - in diesen Zügen hatte sie noch $\mathrm{Rus-}$ tizky charakterisiert - wurde von der Mehrzahl späterer Forscher bestätigt. Wir weisen nur auf die Beobachtungen von Buch, Zahn, Pertik, Kudrewetzky, Herriek und Hektoen, Bozzolo, Rosin, Seegelken, Par kes Weber (1. Fall), Buchstab und Schaposchnikow, Wright, Winkler, Hamburger, Bradshow, Kalischer, MacCallum, Wieland, Jochmann und Sehumm, Harbitz, Bender (2. Fall), A brikos s of f, Saltykow, Vignard und Gallavar din, Ribbert, Sternberg (1904), Menne (2 Fälle), Scheele und Herxheimer, Jellinek, Verebély, Aschoff, Gluzinski und Reichenstein u. a. hin. In sämtlichen hier aufgefïhrten Fällen hatten sich multiple Tumoren im Knochenmark gebildet, die zuweilen so umfangreich 
wurden, daß sie die Knochenschale durchbrachen und in die benachbarten Weichteile hineinwucherten (z. B. im Falle von B u $\mathrm{c} \mathrm{h}$ s $\mathrm{t} a \mathrm{~b}$ ), wie es Sarkome zu tun pfiegen, doch bis zum Ende keine Metastasen gebildet hatten. In dieser Hinsicht ist der von $\mathrm{V}$ e $\mathrm{r}$ e $b$ ély beschriebene Fall sehr interessant: es handelt sich um eine Metastasenbildung im Larynxknorpel, der allerdings vorher eine Verknöcherung und Knochenmarkbildung erlitten hatte. Da die Tumoren in den angeführten Fällen in vielen Knochen gleichzeitig avftraten und nirgends ein primärer Tumor anzunehmen war, so scheint es ganz gerechtfertigt, diese Neubildungen als ,multiple Myelome" zu nennen. Doch begegnèt man unter den zahlreichen beschriebenen Fällen solchen, die sich durch einen großen, anscheinend primär entstandenen Tumor irgend eines Knochens auszeichneten, von dem alle anderen Tumorknoten des Knochenmarks als Metastasen entstanden sein könnten. Derartige Verhältnisse finden wir in den Fällen von $\mathrm{M}$ a r c h a nd (2. Fall, Primärtumor inder Orbita) Bu chst ab und Sch a poschnik of f (Os ilei), Hoffmann (im Darmbein), Lubarsch (im Sternum), Scheele und Herxheimer (im Femur). Scheele und Herxheimer, Parkes Weber (1. Fall) haben sogar auf Anschwellung der dem Primärtumor nächstliegenden Lymphdrüsen hingewiesen, obwohl diese bei typischem Myelom vom Neubildungsproze 3 vollständig verschont bleiben; $\mathrm{H}$ o f f $\mathrm{man} \mathrm{n}$ will Metastasenbildung in der Leber gesehen haben; $\mathrm{L} \mathrm{u} \mathrm{b} \mathrm{a} \mathrm{s} \mathrm{c} \mathrm{h}$, der solche Fälle auch den typischen Myelomen zurechnet, findet für solche Metastasen eine theoretische Berechtigung, indem er überall da Myelomknoten sich bilden läßt, wo dem Knochenmarkgewebe ähnliches Lymphoidgewebe sich vorher vorfand, da das multiple Myelom eine Systemerkrankung des KnochenmarkLymphoidgewebes seiner Meinung nach darstellt, wie zum Beispiel das Lymphosarkom als Systemerkankung des lymphoiden Gewebes der Lymphdrüsen und der adenoiden Körperregionen zu verstehen ist.

Diese theoretischen Überlegungen können natürlich dafür keinen Beweis liefern, daf die Affektionen der Knochen durch sarkomähnliche rundzellige Tumoren, die sogar in die Leber und in die Nieren zu metastasieren fähig sind, zu den Myelomen gehören; allerdings zeigen solche Beobachtungen, wie schwer eine scharfe 
anatomische Grenze zwischen den wahren Myelomen und ihnen höchst nahestehenden Lymphosarkomen der Knochen (des Knochenmarks) zu ziehen ist.

Noch größeres Interesse bieten diejenigen Myelomfälle, welche überhaupt keine Knotenbildung zeigten und als ganz diffuse Knochenmarkaffektion erschienen, wobei sämtliches Knochenmark gewisser Skelettregionen sich in gleichartige Zellenmasse umzuwandeln pflegte. Die Fälle dieser Art verdienen wit vollem Recht zu einer abgesonderten Gruppe „diffuser Myelomatose" der Knochen gerechnet zu werden. Eine solche Art von Knochenmarkaffektion, die schon Macint yre in seinem bekannten Falle beobachtet hatte, beschrieb zum erstenmal $R$ u n e berg, obwohl er sie als Pseudoleukämie gedentet hatte: die Milz war bedeutend vergrößert $(17 \times 11 \mathrm{~cm})$; es bestand starke Anämie, das Knochenmark war nur aus gekörnten blaßgelblichen einkernigen Zellen und aus Riesenzellen zusammengesetzt. Wenn dieser alte Fall nur mit Vorsicht als wahrhafte „Myelomatosis“" zu deuten ist, so entspricht diesem Begriffe vollständig die Beobachtung von March a n d (sein erster Fall). Dieser beschrieb unter dem Namen ,Allgemeine Markhyperplasie mit Schwund der Knochensubstanz" eine bei einem 69jährigen Mann allimählich 'aufgetretene ausges prochene Osteoporose mit Knochenbrüchigkeit und Hyperplasie des Knochenmarks, in dem kleine graue, aus Knochenmarkzellen bertehende Herde sich entwickelt hatten, ohne daß Osteomalazie vorhanden war. Denselben Krankheitstypus sah Senator, doch entwickelte sich hier vor dem Tode Lymphozytose; das Knochenmark. bestand nur aus Lymphozyten; die- Lymphdrüsen waren nicht vergrößert. S enator hat die Hypothese aufgestellt, daß sein Fall eine akute myelogene lymphatische Pseudoleukämie darbot, oder eine an diffuse Myelomatose nahe grenzende Leukämie. Schließlich müssen hierher auch der zweite Fall Parke s We bers, Ell ingers, Sternbergs (1904) und der unserige eingereiht werden (möglicherweise auch die erste Beobachtung von $\mathrm{Seh}$ e ele und Herxheimer, die leider zu kurz skizziert ist). In allen diesen Fällen wurden bei Lebzeiten keine Tumoren notiert; bei der Sektion erwies sich die Affektion des Knochenmarks, seine Umwandlung in Myelom, ganz diffus, nicht knotenförmig, doch von tiefgreifendem Knochenbalkensehwund be- 
gleitet. Eine solche Art von Knochenmarksaffektion unterscheidet sich, - wie S e n a tor mit Recht behauptet hat, wie seinerzeit auch $\mathrm{Runeberg}$ vorausgesetzt hatte und wie später von $\mathrm{S}$ t e r $\mathrm{n}$ b e r g bestätigt wurde, - keineswegs im anatomischen Sinne von der vermutlichen myelogenen Pseudoleukämie, weshalb die diffuse Myelomatose ebenso unmittelbar an diese letzte sich anschließt, wic die entgegengesetzten Vertreter der muitiplen Myelome, die in Form von echten Geschwülsten vorkommen, ohne scharfe Grenze in die Lymphosarkome der Knochen übergehen.

Die Myelome stellen in histologischer Beziehung ebenso großes Interesse wie Mannigfaltigkeit dar. Früher pflegten die Myelomzellen als runde, Sarkomzellen ähnliche Lymphoidzellen beschrieben zu werden. Schon $\mathrm{Rustizky}$ hatte seine Aufmerksamkeit darauf gerichtet, daß die Myelomzellen ihren morphologischen Merkmalen nach mit den normalen Knochenmarkselementen identisch sind; diese Ähnlichkeit wurde zwar von allen Nachforschern bestätigt, doch verfügte man über keine Methode die Myelomzellen von den gewöhnlichen Lymphosarkomzellen oder runden Sarkomzellen zu unterscheiden. Nachdem dann durch Ehrli ch und seine Nachfolger die Methoden der spezifischen Granulafärbung der Blut- und Knochenmarkzellen sich eingebürgert hatten, haben die späteren Forscher, die die Frage der Natur der Myelomzellen zu lösen versuchten, sich bemüht, die morphologische Natur dieser Zellen näher zu präzisieren ( $\mathrm{N}$ e n n e). Es ergab sich, daß die Myelomzellen unter mehreren typischen Formen vorkommen. Am häufigsten bestehen Myelome aus Zellen von runder oder polygonaler Gestalt, wie in unserem Falle, mit einem basophilen gleichmäßig sich färbenden Protoplasma, ohne spezifische Granulierung, und einem Kern, der entweder klein, diffus gefärbt, oder größer und fast bläsehenförmig ist und ein Chromatinnetz und Kernkörperchen besitzt. Diese Zellen differieren keineswegs von demjenigen Typus normaler Knochenmarkzellen, die den Lymphozyten (oder ihren vermutlichen Stanimzellen) histogenetisch am nächsten stehen und sich weiter entwickelnd in ihrem Zelleib Granula ausarbeiten und in typische neutropbile Myelozyten oder einkernige Eosinophile übergehen; solchen noch nicht differenzierten Zellen ist bekanntlich der Name „Myeloblast" gegeben worden. Die 
Mehrzahl der bis jetzt bekannten Myelome bestand aus den hier beschriebenen Zellen.

Neben diesen ,lymphoiden" Myelomen wurden bald auch Myelome anderer Art festgestellt; A s c h of f, Wright, Mac Callum, Hoffmann, Weiß, Verebély, Gluzinski und Reichenstein, $O c c h i$ haben auf die volle Ähnlichkeit der Zellen ihrer Myelomfälle mit U n $n$ a schen Plasmazellen hingewiesen (basophiles Protoplasma mit hellem Hof neben dem Kerne; letzterer exzentrisch gelegen, mit regelmäßig radiär angeordneten Chromatinpartikelchen, sog. „Radkern"). Im Falle von Gluzinski und Reichenstein waren diese Zellen sogar im Blute erschienen. Es kommen also neben ,lymphoiden Myelomen" auch „Plasmozytome" (M a c C a l lu m - L u b a r s c h) oder ,maligne Plasmome" ( $\mathrm{H}$ of f m an $n$ ) vor. Stern berg und $\mathrm{P}$ arkes W eber haben zu diesen zwei Typen noch einen dritten angereiht, dessen Zellen schon als echte reife neutrophile gekörnte Myelozyten erscheinen. Doch umfaßt diese Gruppe ,echter Myelome" nicht nur diejenigen Fälle, wo die neutrophile Granulienung der Tumormyelozyten deutlich ist, sondern auch diejenigen, unserem Falle histologisch identischen, wo die Zugehörigkeit der Tumorzellen zu den Myeloblasten außer Zweifel ist. Wie Gluzinski und Reichenstein in der zweiten Gruppe, so beschrieb $\mathrm{S}$ ternberg in dieser letzten ein Myelom (Chloromyelosarkom) mit massenhaftem Übertritt der neutrophilen Myelozyten in den Blutstrom, wodurch das klinische Bild myelogener Leukämie vorgetäuscht wurde.

Schließlich bleibt bis jetat noch isoliert da stehen die Beobachtung $\mathrm{Ribberts:}$ in seinem Falle multipler knotiger Knochenmarkhyperplasien bestanden die Tumoren aus hämoglobinhaltigen, normalen Erythroblasten des Knochenmarks identischen Zellen. Deshalb schlug R ib b ert vor diese Geschwulst „Erythroblastom"zu nennen. Neuere derartige Publikationen fehlen.

Indem wir alles oben Gesagte kurz zusammenfassen, möchten wir als dem Stande der Tatsachen am genauesten entsprechend die von $\mathrm{Sternberg-Lubarseh}$ vorgeschlagene Klassifikation unterstützen, die die Myelome zu

1. Lymphozytomen (diese schließen sich den I,ymphosarkomen des Knochenmarks an), 
2. Myelomen,

3. Plasmozytomen (malignen Plasmomen $\mathrm{H}$ of $\mathrm{f} \mathrm{m}$ a $\mathrm{n} \mathrm{n} \mathrm{s}$ ) und

4. Erythroblastomen ( Rib b e r t s) zerfallen läßt.

Das eingehende Studium verschiedener Myelomarten zeigt, daß man zuweilen solchen Fällen begegnet (vgl. die Fälle von S t e r n b e r g, S e n a t or u. a.), die so gut wie gar nicht in den angedeuteten Rahmen passen, dagegen als Ủbergangsformen dastehen und ebenso gut dem Begriffe Leukämie, Pseudoleukäniie oder Lymphosarkome entsprechen. Der Unbestimmtheit unserer Kenntnisse über die hyperplastischen Prozesse des Knochenmarks halber darf es nicht überflïssig erscheinen, hier eine andere überhaupt alle das Knochenmark berührenden hyperplastischen Prozesse umfassende Klassifikation, die von $\mathrm{P}$ a r k s W e ber (a. a. $O$. S. 430) vorgeschlagen wurde, anzuführen.

a) Hyperplasie der lymphoiden Zellen des Knochenmarks, die in den Blutstrom nicht übergehen: myelogene Pseudoleukämie, myelogenes Lymphosarkom, Lymphadenomatosis ossium ( $\mathrm{N}$ o t h n a ge l), multiples Myelom und Myelomatosel lymphoiden Typus.

b) Id., doch überschwemmen die Lymphozyten den Blutstrom: sog. akute myelogene Leukämie (Fälle von D en nig, Münch. med. Wochenschr. 1901, und von M e ll a n d, Medical Chronicle, sept. 1902). Die anderen lymphoiden Organe nicht affiziert (Fall von Se n a t o r ).

c) Hyperplasie der Lymphozyten der Lymphdrüsen und anderer lymphadenoiden Gewebe; keine Lymphozyten: Ho d g $\mathrm{k}$ i n e s Pseudoleukämie.

d) Id., doch mit Lymphozytose: lymphatische ind splenogene Leukämie.

e) Hyperplasie der Knochenmarkmyelozyten, die in den Blutstrom nicht übergehen: myelogene Pseudoleukämie-= Myelomatose des myelozytären. Typus (immer von Albumosurie begleitet),

f) Id., doch überschwemmen die Myelozyten das Blut; keine Albumosurie: myelogene oder spleno-myelogene Leukämie.

- Zur Vervollkommnung der anatomischen Charakteristik des multiplen Myeloms ist noch auf eine in allen Fällen beobachtete 
Eigenschaft hinzuweisen, nämlich auf seine Fähigkeit, schnell die Knochenspongiosa zu resorbieren und Osteoporose hervorzurufen. Dieses Anzeichen ist so konstant und wesentlich, daß das Ausbleiben der Osteoporose und um so mehr eine Osteosklerose oder Osteophytenbildung, die z. B. Noth $\mathrm{n}$ a g e $\mathrm{l}$ und $\mathrm{H}$ am mer beobachtet hatten, einen genügenden Grund geben muß, um die von diesen Forschern beschriebenen Tumorbildungen aus der Gruppe der Myelome auszustreichen und einer anderen Gruppe multipler sklerosierender Lympho- oder Osteosarkome einzureihen.

Die Knochenresorption bei Myelomen weicht ebenso von derjenigen bei Osteomalazie, wie bei anderen Fällen des Knochensehwundes (Knochenatrophie), z. B. im hohen Alter, rings um andere Tumoren usw., wesentlich ab. Die Knochenresorption bei Myelomen wird namentlich nicht durch Osteoklasten $\mathrm{R}$ ob in s bewirkt, wie es bei Knochenschwund aus anderen Ursachen zu geschehen pflegt, sondern durch die Tumormassen der Myelome selbst, welche die Knochenbalken rings umschließen und deren schnelle Einschmelzung und Schwund verursachen. Die Halisterese geht dabei mit Schwund der weichen Knochengrundsubstanz selbst Hand in Hand, indem diese letzte bei Osteomalazie ungeachtet der Beraubung der Kalksalze zurückbleibt und sogar neues Osteoidgewebe gebildet wird. Dank dem Parallelismus dieser zwei Prozesse bei Myelomen werden die Rippen z. B. brüchig, spröde, doeh nicht weich. Auf das Fehlen von Osteoklasten bei Myelombildung, sowie deren untergeordnete Rolle bei dieser Art von Osteoporose wurde schon von seiten mehrerer Autoren hingewiesen ( $B$ u ch, Winkler, Bender, A brikos soff, Saltykow, Parkes Weber, Scheele und Herxheimer, Collins, Verebély u. a.), und es mag als zweifellos festgestellt angesehen werden, daßo die Myelomzellen eine eigene knochenresorbierende Eigenschait besitzen.

Die Osteoporose bedingt, wie oben erwähnt wurde, Knochenbrüchigkeit der Rumpfknochen, welche sich durch sehr leicht eintretende Brüche der Rippen, Schlüsselbeine, ja sogar zuweilen der Oberschenkelknochen kennzeichnet, und kann als erstes Anzeichen einer Knochenerkrankung auf das Vorhandensein einer Myelomatose die Aufmerksamkeit lenken. Seitens der Wirbelsäule äußert sich die Knochenporose durch Deformierung, Krümmung und Verkürzung des Rumpfes und Brustkorbes, was aus- 
führlich von $\mathrm{Parkes} W$ eber in seinem schönen und erschöpfenden Werke (Fälle von W e b e r und. Brads how) geschildert und illustriert worden ist. Doch außer ihrer Wirkung auf das Skelett, kann die Knocheneinschmelzung durch Myelombildung, wenn sie stürmisch vor sich geht, eine so reichliche Menge von Kalksalzen aus den Knochen auslösen, daßj die letzten aus dem Organismus sich durch den Harn und Kot abzusondern nicht Zeit genug haben und in anderen Organen sich ablagern. Ein derartiges Ereignis fand in unserem Falle satt. Obwohl die Myelomatose noch keine Einbrïche der Rippen bewirkt hatte, ging doch der Wuchs der Neubildung so schnell und extensiv vor sich, daß eine massenhafte Kalkmetastase im Sinne Virchows eintrat.

Derartige Organverkalkungen, zwar in viel bescheidenerem Maße, sind schon mehrmals in der Literatur erwähnt worden, nämlich bei Tumoren der Knochen, eitriger Osteomyelitis, speziell auch bei multiplen Myelomen. Vir e ho 0 w war der erste, der auf Kalkablagerungen hauptsächlich in den Alveolensepten der Lungen, zwischen den Drüsen der Magenschleimhaut, in den Membranae propriae der Nierenkanälchen und im Mastdarm hinwies, und als Ursache dieser Ablagerungen die Einschmelzung der Knochen durch Eiterprozesse und Neubildungen angab. Nach Vir chow beschrieben Lungenverkalkungen $\mathrm{Ch}$ i a ri, Hlava, Verkalkung der Dickdarmschleimhaut - Grohe (alle diese drei Fälle boten dazu keine Ursache seitens des Skeletts); Kü t $t \mathrm{n}$ e r hat eine Kalkmetastase in die peripherischen Arterien bei eitriger Entzündung der Wirbel gesehen; Hascovec-Kalkablagerungen in den Lungen, Nieren (in den Glomeruli und interstitiell); $\mathrm{D}$ a vi ids o h $\mathrm{n}$ beobachtete bei einem Sarkom der Beckenknochen und der Wirbelsäule Kalkablagerungen in den Lungen, Nieren, im Magen und in den Herzklappen. K o c k e l beschrieb 6 Fälle von Lungenverkalkung bei Knochenkarzinom, Emphysem und Herzfehlern. IK i s ch en sk y beschrieb umfangreiche Ablagerungen von phosphorsaurem Kalk in den Lungen mit katarrhalischer Pneumonie und in der Magenschleimbaut in den Kapillaren und Membranae propriae; auf eine mikroskopische Untersuchung der Knochen wurde leider verzichtet. $B$ ender notiert reichliche Kalkablagerungen in den Alveolensepten und Gefäßen der Lungen und in dermittleren Schicht der Magenschleimhaut, in den Gefäßen der Submukosa, in den elastischen Fasern der Muskularis, in den Membranae propriae der Nierenkanälehen und innerhalb derselben, teils in den Glomeruli bei einem 14jährigen Knaben, der einem multiplen Knochensarkom erlag.

Die Literatur über Myelome enthält ebenso einige Erwähnungen über Kalkablagerungen in Lungen und Nieren; so sah derselbe B e n d e r (2. Fall) Kalkablagerungen, die an die von uns beschriebenen Bilder, zwar in geringerem Mabstabe, erinnern: die Kalkniederschläge fanden sich in den Alveolensepten in Gefäßwänden, welche in Kalk röhren sich umgewandelt hatten, die Alveolen enthielten dabei junges Granulationsgewebe; doch waren die kalkhaltigen Partien der Lunge nicht zahlreich. Parkes Weber hat in seinem ersten 
Myelomfalle Kalkablagerungen in den Nieren (und Lungen?) notiort, S c h e e le und Herxheimer in und zwischen den Nierenkanälchen.

Alle die erwähnten Fälle können kaum mit den kolossalen von uns geschilderten Kalkablagerungen hauptsächlich in den Lungen verglichen werden: die Kalkdurchtränkung des Lungengewebes war so groß, daß die Atemzüge - Aufblähung und Zusammensinken - so gut wie ummöglich geworden sind und die Kranke in schwere Asphyxie verfiel. Eine so reichliche Kalkablagerung findet in der stürmischen. Knocheneinschmelzung der von Myelomatose angegriffenen Skelettpartien eine befriedigende Erklärung.

Um eine gewisse Vorstellung über die in den Weichteilen auf Kosten der Knochenhalisterese angesammelten Kalkmengen zu gewinnen, wurden Analysen verschiedener Organe auf Wasser, trockenen Rückstand, Asche, Kalk und teils auf Phosphor ausgeführt (Dr. Helene Kolessnikoff), deren Resultate wir hierin Tabellen zusammengestellt und mit anderen Analysen verglichen angeben.

Tabelle I. Rippe.

\begin{tabular}{|c|c|c|c|c|c|c|}
\hline$\%$ & 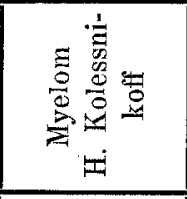 & 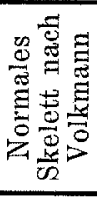 & 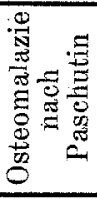 & 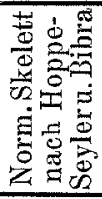 & 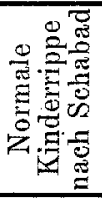 & 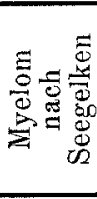 \\
\hline Wasser & 58,8 & 34 & 50 & -1 & 一 & - \\
\hline Trock. Rückst. & 41,2 & - & 一 & - & 一 & - \\
\hline Asche & $\left.23,19(56,28)^{1}\right)$ & - & - & $35(65)$ & - & - \\
\hline $\mathrm{Ca} O$ & $12,09(29.34)$ & - & - & - & 25,28 & 15,39 \\
\hline $\mathrm{P}_{2} \mathrm{O}_{5}$ & $9,43 \quad(22,88)$ & - & - & - & - & 18,0 \\
\hline
\end{tabular}

Tabelle II. Manubrium sterni.

\begin{tabular}{|c|c|c|c|c|}
\hline$\%$ & 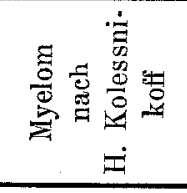 & 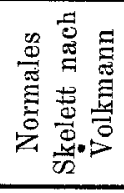 & 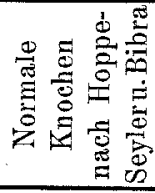 & 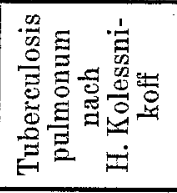 \\
\hline Wasser & 73,9 & 34 & - & 62,67 \\
\hline Trock. Rückst. & 26,1 & - & 一 & 37,33 \\
\hline Asche & $\left.8,34(31,87)^{1}\right)$ & - & $35(65)$ & $16,44(44,03)$ \\
\hline $\mathrm{Ca} 0$ & $4,14(15,8)$ & 一 & - & $9,09(24,08)$ \\
\hline $\mathrm{P}_{2} \mathrm{O}_{5}$ & $3,9(14,9)$ & - & - & $6,9(18,5)$ \\
\hline
\end{tabular}

1) Die eingeklammerten Zahlen stellen die zum trockenen Rückstand berechneten \% vor. 
Tabelle III. Lunge.

\begin{tabular}{|c|c|c|c|c|c|}
\hline$\%$ & 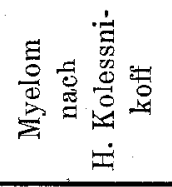 & 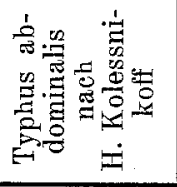 & 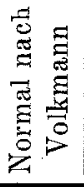 & 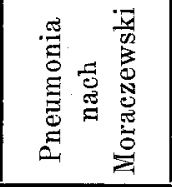 & 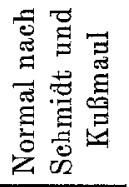 \\
\hline & 80 , & 84,91 & 78 & - & - $\cdot$ \\
\hline rock. Rückst. & 19,42 & 15,09 & - & - & 一 \\
\hline Asche & $\left.7,3(37,59)^{1}\right)$ & $0,701(4,64)$ & 一 & 一 & $(2-6,68)$ \\
\hline $\mathrm{Ca} O$ & $2,6(13,38)$ & $0,006(0,039)$ & 一 & $0,004(0,033)$ & - \\
\hline $\mathrm{P}$ & $2,04(10,5)$ & $0,231(1,53)$ & - & $0,188(\mathbf{1}, 51)$ & - \\
\hline
\end{tabular}

Tabelle IV. Niere.

\begin{tabular}{|c|c|c|c|c|}
\hline$\%$ & 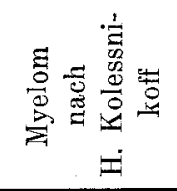 & 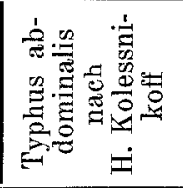 & 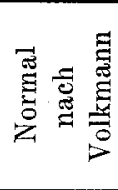 & 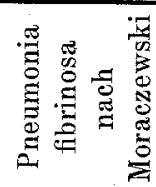 \\
\hline Wasser & 85,19 & 78,9 & 77 & - \\
\hline Trock. Rückst. & 14.81 & 21,1 & - & - \\
\hline Asche & $\left.1,8(12,15)^{1}\right)$ & $1.04(4,9)$ & - & - \\
\hline $\mathrm{CaO}$ & $0,41(2,76)$ & $0,002(0,009)$ & - & $0,003(0,03)$ \\
\hline
\end{tabular}

Tabelle V. Aorta.

\begin{tabular}{|c|c|c|}
\hline$\%$ & 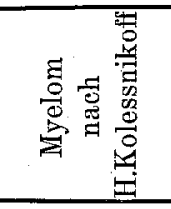 & 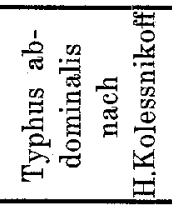 \\
\hline & 68,2 & 78,5 \\
\hline Trock. Rückst. & 31,8 & 21,5 \\
\hline Asche & $\left.0,509(1, \tilde{0})^{1}\right)$ & $0,88(4,09)$ \\
\hline & $0,12(0,37)$ & $0,16(0,74)$ \\
\hline $\mathrm{P}_{2} \mathrm{O}_{5}$ & $0,135(0,42)$ & $0,006(0,027)$ \\
\hline
\end{tabular}

Diese Zahlen zeigen, daß die Knochen in hohem Maße ihres Kalks beraubt sind, der hauptsächlich in die Lungen (größere Zahlen der Kalkmenge finden sich nur im Falle $\mathrm{Ch}$ i a $\mathrm{r}$ is - bis

1) Die eingeklammerten Zahlen stellen die zum trockenen Rückstand berechneten $\%$ vor. 
$45,53 \%$ Asche nach B a m b erg e r), in den Magen (auf dessen Analyse wurde leider verzichtet), in die Därme, Nieren, in die Milz und in die Gefäße anderer Organe transportiert wurde.

Was die Ursachen der vorzüglich in den Lungen und der Magenschleimhaut stattgehabten Kalkablagerungen anbetrifft, so muß diese Frage offen bleiben; es liegt nur eine Vermutung nahe, daß die Kalksalze, mit denen im Knochensystem das Blut sich angereichert hat, sofort die Neigung äußern, auszufallen, nachdem der Blutstrom das Herz passiert hat, weswegen der kleine Kreislauf als Speicher dienen muB, um so mehr als das Blut in den. Lungen schnell seine Kohlensäure verliert und mehr alkalisch wird; um dieselbe Reaktionsänderung handelt es sich in der Magenschleimhaut bei Abscheidung freier Salzsäure. Die Gefäße, welche sich mit dem mit Kalksalzen gesättigten Blute unmittelbar berühren, (nämlich Arterien) müssen den Kalkinkrustationen in erster Linie Platz geben, was auch tatsächlich geschehen ist. Hier ist es interessant anzufügen, daß die Aorta, wie aus der Tabelle $V$ hervorgeht, eine Ausnahme von der Regel darbot und weniger kalkhaltig war, als eine zur Kontrollanalyse bei einer Typhuskranken (desselben Alters) aufgehobene.

Es wurde schon mehrmals erwähnt, daß typisches multiples Myelom sich durch B en c e-J o h n es sche Albumosurie dokumentiert. Auf die große differentiell-diagnostische Bedeutung dieses Symptoms zum Unterschied der Myelomatose von der Osteomalazie wurde noch von $\mathrm{Kahler}$ hingewiesen; später wurde diese Tatsache seitens vieler Forscher bestätigt ( $\mathrm{Ka}$ lischer, Se e gelken, Buchstabund Schaposchni koff, Jochmann und Schumm, Verebély, Gluzinski und Reichenstein u. a.); ja sogar oft zur Stellung der Diagnose auf Myelom bei Lebzeiten benutzt ( Rosin, Wright, Ellinger, Buchstab und Schaposehnikoff, Bozzolo, Paget Moffat, Barr, Bradshow, Hamburger (2 Fälle), Parkes W e ber, D o n e t ti

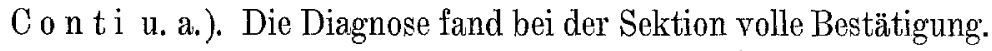

Eine Zusammenfassung aller Fälle konstanter Albumosurie, welche von $B$ u chst a b und $S \mathrm{ch}$ a posehnik off, Parkes Weber, Paget Moffat, Askanazy u. a. unternommen wurde, hat zum Schlusse geführt, daß reichliche Albumosurie fast 
stets auf Myelomatose hinweist, und daß in dem Falle, wo sie während der ganzen Krankheit wirklich ausbleibt, die Affektion des Knochenmarks dureh Myelome zweifelhaft sein darf (siehe den Fall von Collins z. B.); wenn dagegen Albumose in großen Mengen ausgeschieden wird, so ist mit vollem Recht eine diffuse pathologische Hyperplasie des Knochenmarks oder Tumorbildungen zu vermuten, selbst wenn während des Lebens Symptome einer anderen Erkrankung in den Vordergrund getreten wären.

Zu derartigen Fällen gehören: dic Beobachtung Aska n a z y s: lymphatische Leukämie myelogenen Ursprungs, doch mit Knochenmarkhyperplasie, die zu Osteoporose und Rippenbrüchen geführt hatte; dann ein Fall von Myxödem, der von F i I z beschrieben worden ist. In unserem Falle wurde Myelomatose von zweifelloser Albumosurie begleitet, die, wäre ihr zur rechten Zeit gebührende Aufmerksamkeit geschenkt worden; zum Ausgangspunkte einer frühen und sicheren Diagnose hätte dienen können, nämlich in einer Krankheitsperiode, wo weder objektive noch subjektive Symptome eine Knochenaffektion zu vermuten rechtfertigten.

\section{Schlu B f olg erungen.}

1. Unter dem Namen Myelom des Knochenmarks wird eine multipel auftretende Hyperplasie irgendeines Zelltypus der Knochenmarkelemente verstanden, wobei alle anderen Knochenmarkzellen durch diesen in Vermehrung begriffenen Zelltypus verdrängt werden. Die Myelome geben nie Metastasen in andere Organe.

2. Die Myelome bilden sich in den Rumpfknochen (Brustbein, Rippen, Claviculae, Wirbel, Becken, Schulterblatt, Schädel, selten im Oberarm und Oberschenkelknochen) entweder in Form knotiger Geschwülste, oder diffus. Die ersten grenzen an gewöhnliche, Lymphosarkome an, die letzten an Knochenmarkhyperplasien bei myelogenen Leukämien (und Pseudoleukämien).

3. In histologischem Sinne zerfallen die Myelome in a) Lymphozytome, b) Myelome, c) Plasmozytome oder Plasmome und d) Erythroblastome.

4. Myelome besitzen stets die Fähigkeit den Spongiosaknochen einzuschmelzen; dieser Schwund der Spongiosa wird zuweilen von Kalkmetastasen in die Lubgen, den Magen, die Nieren, die Därme und andere Organe begleitet. 
5. Echte Myelome gehen stets (mit wenigen Ausnahmen) mit $\mathrm{B}$ e $\mathrm{n} \mathrm{c}$ e - $\mathrm{J}_{0} \mathrm{~h} \mathrm{n}$ e s scher. Albumosurie Hand in Hand und haben darin ihren differentiell-diagnostischen Unterschied von der Osteomalazie und von den Systemerkrankungen des Blutes und der blutbildenden Organe.

6. Die Myelome befallen ältere Subjekte, viel öfter Männer und führen stets zu mehr oder weniger starker sekundärer Anämie.

\section{Literatur.}

Außer der bei J e $1 \mathrm{l}$ i n e $\mathrm{k}, \mathrm{Z}$. klin. Diagn. u. pathol. Anat. d. multiplen Myeloms in Virehows Archiv Bd. 177 S.' 96, 1904 angegebenenLiteratur ist noch die nachstehend angegebene zu beachten.

A s c h of f, Ein Fall von Myelom. Münch. med. Wschr. 1906, S. 337. A s k a n a $7 \mathrm{y}$, Üb. d. diagnost. Bedeut. d. Ausscheidung d. B e n e e-J o h n e sschen Körpers durch d. Harn. D. Arch. f. klin. Med. Bd. 68, 1900, S. 34. B e chtold, Üb. d. multiple Myelom. I.-Diss., Würzburg 1902. - B u c h stabu. Schaposchnik of f. Diffus. Myelom d. Rumpfknoch. nebst typ. Albumosurie als charakterist. diagnost. Symptom. Russ. Arch. f. Path. 1899 (Russisch). — Collins, Multiple Myeloma. Med. Record 67, 1905, S. 641. - C onti, Albumosuria e neoplasie sistemat. delle Ossa. La Clin. med. Ital. 1902, p. 211-247; zit. nach Parkes Weber. - Davids o h n, Fragmentation d. elast. Fasern. Virch. Arch. Bd. 160, 1900, S. 538. D i ck, A case of multiple Myeloma. Transact. of the Chicago path. Soc. 6, 1904, S. 168. - D o e ring, Beitr. z. Lehre v. d. idiopath. Osteopsatyrosis. D. Zitschr. f. Chir. Bd. 77, 1905, S. 284 . - D o n e t ti, Sulla malattia di $\mathrm{K}$ a h $\mathrm{l}$ e r. Riv. Crit. di Clin. Med. 1901, S. 789; zit. nach P a r ke s W e ber. - Funkenstein. Ein Fall von multiplem Myelom. I.-Diss., Straßburg 1900. - G l u zins ki u. R e i chen s te in, Hyeloma u. Leucaemia lymphat. plasmocellul. Wien. klin. Wschr, 1905, S. 386. - G r o he e, U̇b. Kalkmetastase. Virch. Arch. Bd. 13 S. 277. - G r o s c h, Multiple Myelome d. Schädaldaches. I.-Diss., München 1905. - $\mathrm{Ha} \mathrm{mburger}$, Two exemples of B enceJohnes' Albumosuria assoc. with multiple Myeloma. John $\mathrm{H}$ o p k in s Hosp. Bull. XII, 1901. S. 38. - H a s c ove c, Sur la calcification endoyen des poumons. Arch. bohème IV, H. 3. - H elly, K., D. hämatopoet. Organe in ihren Bezieh. z. Pathol. d. Blutes. Wien 1906. - - H e r r i ck and Hekt o e n. Myeloma; report of a case. Med. News 1894, S. 239. - H lava, Z. Ätiol. d. Lungenverkalk. Wien. med. Blätt. 1882, Nr. 36-38. - H o f f ma $n \mathrm{n}$, Üb. d. Myelom mi. bes. Berücksicht. d. malignen Plasmoms. Z i e glers Beitr. Bd. 35 S. 317. - Derse 1 b e, Üb. Myelomatose, Leukämie u. Ho d gk in sche Krankh. Arch f. klin. Chir. Bd. 79, 1906, S. 384. - K al i seher, Ein Fall v. Ausscheid. d. Bence-Johnes schen Eiweißkörp. durch d. Urin b. Rippenmyelomen. D. med. Wschr. Bd. 27, 1901. - Kis chensky, Utb. Kalkablag. i. d. Langen u. i. Magen. Mediz. Obozrenie Bd. 54, 1900 , S. 849 (russisch). - K o c ke l, Ủb. d. Kalkinkrustation d. Lungengew. D. Arch. f. klin. Med. Bd. 64, 1899. - K ü ttn e r, Ein Fall v. Kalkmetastase. Virch. Arch. Bd. 55, 1872. - L u b a r s c h, Z. Myelomfrage. Virch. Arch. Bd. 184 S. 213. - M a r c h a n d, Fall v. allgem. Markhyperplasie m. Schwind d. Knochensubst. D. med. Wschr. 1886, S. 486. Menne, Z. Kenntn. d. Myelomzellen. Virch. Arch. Bd. 183 S.115. Oc chi, C. degli, Multiple Sarkomatose d. Knochen. Mailand 1907. Ref. D. med. Wsehr. 1908, S. 1201. - P a g e $t$ M of f a t, Myelopathic Albumo- 
suria. The Lancet 1905, S. 207. - Q u a cken bos s, Multiple Myeloma with involvement of the orbit. Journ. of med. res., 15, 1906, S. 261. - R u n e b er g, Ein Fall v. medull. Pseudoleukämie. D. Arch. f. klin. Med. Bd. 33, 1883. - Scheele u. Her x he i m e r, Utb. cinen bemerkenswert. Fall v. multiplem Myelom. Ztschr. f. klin. Med. Bd. 54, 1904, S. 57. - S e n a t o r, Üb. lymphadenoide u. aplast. Veränder. d. Knochenmarks. Ztschr. f. klin. Med. Bd. 54, 1904. - S o r g e, Utb. einen Fall v. B e n e e - J o h n e s schem Körp. b. Erkrank. d. Rumpfskeletts. I.-Diss., Jena 1900. - S p i e ge l. b e r g, Beitr. z. Kenntn. d. multipel anftret. Knochensarkome. J.-Diss. Freiburg 1894. - Sternberg, Z. Kenntn. d. Chloroms. Z i e glers Beitr. Bd 37, 1905, S. 437. — S ü s s m a n n , Ủb. einen neuen Fall v. multipler Myelombild. verbund. m. hochgrad. Albumosurie. I.-Diss., Berlin 1897. - V e r e b él y, Üb. d. Myelom. Beitr. z. klin. Chir. Bd. 48, 1906, S. 614. - Vi g $\mathrm{n}$ a $\mathrm{rd}$ d t G a llaverd in, Du myélome multiple des os avec albumosurie. Rev. de chir. 27, 1903, S. 91. - Vir c h ow, Kalkmetastasen. Virch. Arch. Bd. 8, 1855, S. 103. - D e r s el b e, Kalkmetastasen. Virch. Arch. Bd. 9, 1856. W p i s s, Ủb. einen Fall v. Myelom d. Darmbeins m. Metastasenbild. I.-Diss. München 1905 .

\section{VIII.}

\section{Über einen Fall von akuter großzelliger Jymphatischer Leukämie mit generalisierter Hanterkrankung.}

(Aus der Dermatologischen Klinik und aus dem Pathologischen Institute der

Universität Bern.)

Von

Frau Dr. med. Rahel Rodler-Zipkin (Nürnberg), gewes. IT. Assistentin am Pathologischen Institute und Volontärassistentin an der Dermatologischen Klinik der Universität Bern.

Obschon seit der Entdeckung der Lenkämie durch V i r c h o w (1845) mehr als 60 Jahre verflossen sind, blieben einzelne Fragen in der Pathologie dieser Krankheit noch bis zur Stunde strittig.

Ist auch die Auffassung der Leukämie als einer primären parenchymatösen Bluterkrankung, wie sie B i esi a d e cki und L öw i t gehabt haben, als altgemein verlassen zu betrachten, so herrscht doch noch keine vollständige Einigkeit unter denjenigen Autoren, die den Schwerpunkt der Leukämie in die Veränderungen der hämatopoetischen Organe verlegen. Es mehren sich die Fälle, die in den Rahmen der von Ehrlich-Pink us einerseits, $\mathrm{Ne}$ u $\mathrm{m}$ a $\mathrm{n}$ - $\mathrm{P}$ a p p e $\mathrm{n}$ h e i m andererseits aufgestellten Theorie der Leukämie nicht ganz hineinpassen. Das veranlaßte $\mathrm{S}$ t e r n - 